\title{
Distribuição espacial da obesidade em área urbana no Brasil
}

\author{
Spatial distribution of obesity in an urban Brazilian area
}

Fernanda Penido Matozinhos ${ }^{1}$
Crizian Saar Gomes ${ }^{1}$
Marcelo Azevedo Costa ${ }^{2}$
Larissa Loures Mendes $^{3}$
Milene Cristine Pessoa
${ }^{4}$
Gustavo Velasquez-Melendez

Abstract The spatial distribution of a disease is important for the diagnosis and epidemiological understanding of the health situation and trends, enabling a better grasp of the factors that determine the health status of populations. The scope of the study was to analyze the spatial distribution of obesity in adults in Belo Horizonte. This cross-sectional study was developed by Telephone Survey from the database for 2008 to 2010 of the Surveillance of Risk and Protective Factors for Chronic Diseases. Obesity was defined as body mass index $30 \mathrm{~kg} / \mathrm{m} 2$. A georeferenced base with the environmental data of addresses and zip codes of the location was developed and spatial scan statistics were employed. A comparative analysis of environmental variables related to clusters of higher and lower prevalence of obesity was conducted. A cluster of obese individuals without statistical significance was found in the central area of the city. Also, a significant cluster of non-obese individuals was found in the eastern area of the city. These findings suggest that reasons for low prevalence of obesity in urban Brazilian areas could be related to better social organization and high availability of places for food stores and the practice of physical activity.

Key words Spatial analysis, Obesity, Public health
Resumo A distribuição espacial de uma doença é importante para o diagnóstico e o conhecimento epidemiológico da situação e das tendências de saúde, permitindo uma melhor compreensão acerca dos fatores que determinam o estado de saúde das populações. O objetivo do estudo foi analisar a distribuição espacial da obesidade em adultos em Belo Horizonte, a partir da base de dados do Sistema de Vigilância de Fatores de Risco e de Proteção para Doenças Crônicas por Inquérito Telefônico de 2008 a 2010. A obesidade foi definida como índice de massa corporal $30 \mathrm{~kg} / \mathrm{m} 2$. Foi desenvolvida uma base georreferenciada com os dados ambientais a partir do endereço e CEP do local. Empregou-se a estatística de varredura espacial. Uma análise comparativa das variáveis ambientais referentes aos conglomerados de maior e menor prevalência de obesidade foi realizada. Foi encontrado um conglomerado de indivíduos obesos na área central da cidade, sem significância estatística. Verificou-se, também, agrupamento significativo de indivíduos não obesos no leste da cidade. Esses resultados sugerem que as razões para a baixa prevalência de obesidade em áreas urbanas brasileiras podem estar relacionadas à melhor organização social e alta disponibilidade de comércios de alimentos e de locais para a prática de atividade física.

Palavras-chave Análise espacial, Obesidade, Saúde pública 


\section{Introdução}

Nos últimos anos, a obesidade tem sido estudada a partir de uma perspectiva ecológica' ${ }^{1}$ que considera o ambiente em que a pessoa vive como fator associado para a prevalência deste agravo. De fato, as características ambientais podem influenciar em diversos aspectos, dentre eles as escolhas de hábitos de vida, relacionados principalmente à alimentação e à prática de atividade física, o que pode favorecer o aumento da obesidade ${ }^{2}$.

Estudos mostram que existem diferenças dos fatores ambientais obesogênicos entre os países e, também, variações espaciais dentro de um mesmo país pelo fato de a distribuição geográfica ser desigual, mesmo em escala local. Além disso, verifica-se que a prevalência da obesidade pode variar geograficamente por inúmeras razões, dentre elas fatores comportamentais (como o consumo inadequado de alimentos e prática da atividade física insuficiente), composição genética da população e variações do ambiente e $^{3-9}$.

Dessa forma, para propor intervenções inovadoras e eficientes, é importante avaliar a distribuição de variáveis relacionadas ao ambiente e que podem influenciar padrões de atividade física, de consumo alimentar e o ganho de peso ${ }^{10}$. O conhecimento da distribuição espacial (conglomerados ou clusters espaciais) de obesidade é uma das questões essenciais para o diagnóstico e conhecimento da situação epidemiológica da saúde das populações e das suas tendências e pode indicar uma necessidade de definição de ações de planejamento urbano direcionadas a grupos de determinada área geográfica, uma vez que a "epidemia de obesidade" pode ter um padrão temporal e espacial bem definidos, ${ }^{9} 11-13$.

Ressalta-se que, apesar do seu grande potencial e das suas aplicações em inúmeras áreas do conhecimento, as técnicas de análise espacial ainda são limitadas na área da saúde, especialmente em países em desenvolvimento, em virtude das dificuldades inerentes à manipulação de dados geográficos?.

Poucos são os trabalhos com análises espaciais que consideram as variações geográficas em nível local (ou seja, utilizam códigos postais) e há uma escassez de pesquisas abordando análise espacial da obesidade em países em desenvolvimento, especialmente no Brasil. Estudos sugerem que as áreas geográficas de menores dimensões podem oferecer novas contribuições a respeito dos determinantes espaciais de saúde ${ }^{14,15}$. Neste sentido, o objetivo do presente estudo foi analisar a distribuição espacial da obesidade em adultos, de 2008 a 2010, no município de Belo Horizonte, Minas Gerais.

\section{Métodos}

Trata-se de estudo epidemiológico transversal, desenvolvido a partir da base de dados do Sistema de Vigilância de Fatores de Risco e de Proteção para Doenças Crônicas por Inquérito Telefônico (VIGITEL), utilizando as amostras de Belo Horizonte referentes aos anos de 2008 a 2010.

A amostra final foi constituída por 5273 indivíduos. A Figura 1 refere-se ao fluxograma das perdas amostrais. Realizou-se uma análise comparativa das perdas e não foram verificadas diferenças significativas da amostra final.

A obesidade foi definida como Índice de Massa Corporal (IMC) $\geq 30 \mathrm{~kg} / \mathrm{m}^{2}$, critério proposto pela Organização Mundial de Saúde ${ }^{16}$. O IMC foi obtido a partir da relação [peso $(\mathrm{kg}) /$ altura $^{2}(\mathrm{~m})$ ] e calculado a partir do peso e da altura autorreferidos.

Para caracterizar o ambiente, foi desenvolvida uma base geocodificada com os dados ambientais (comércios de alimentos - restaurantes,

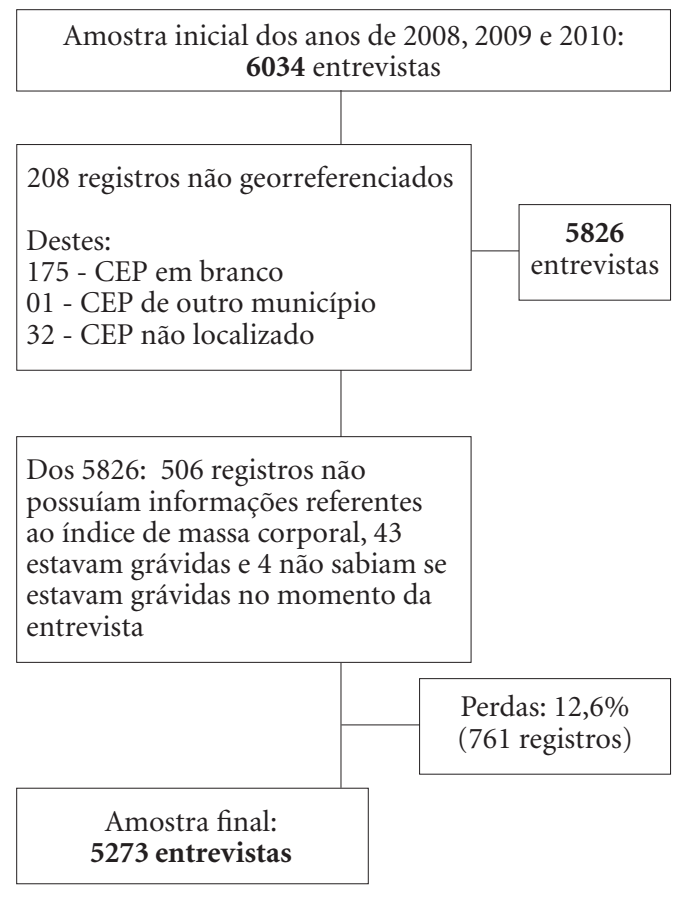

Figura 1. Fluxograma das perdas amostrais 
lanchonetes, bares, vendedores ambulantes, supermercados, hipermercados e estabelecimentos especializados na venda de frutas e hortaliças-; locais públicos e privados para prática de atividade física; densidade residencial; taxa de homicídios e renda da vizinhança), à qual foram incorporados os dados individuais de cada participante da amostra.

Esses dados foram georreferenciados a partir do endereço e CEP do local, obtidos a partir de diversas fontes comerciais e governamentais, como: Classificação Nacional de Atividades Econômicas (CNAE), Secretaria Municipal Adjunta de Segurança Alimentar e Nutricional (SMASAN), Centro Integrado de Informações de Defesa Social (CINDS) da Polícia Militar de Minas Gerais, Instituto Brasileiro de Geografia e Estatística (IBGE), Secretaria Municipal de Saúde (SMS) e Empresa de Informática e Informação do Município de Belo Horizonte S/A (PRODABEL).

Foi realizada a distribuição espacial da obesidade de acordo com as áreas de abrangência das Unidades Básicas de Saúde (AAUBS), que são uma das formas de organização do sistema público de ações e políticas públicas de saúde. Para verificar a presença de conglomerados de alta ou baixa prevalência de obesidade, foi utilizada a técnica de varredura espacial ${ }^{17}$, também conhecida como estatística de varredura espacial scan. Esse método é utilizado para identificar agrupamentos para diferentes raios de varredura e é testada a hipótese nula de que os agrupamentos ocorram aleatoriamente na região de estudo. Define-se buffers como um conjunto de círculos cujos centros são os pontos georreferenciados e os raios aumentam gradativamente, de forma que os menores círculos contenham um único ponto e os maiores círculos contenham 50\% dos pontos. Dentre o conjunto de círculos (buffers), foram avaliados os que apresentaram a maior e a menor prevalência da obesidade em relação aos demais $^{17}$.

O nível de significância adotado foi $=0,05 \mathrm{e}$ as análises foram realizadas com o uso do Software SaTScan versão 9.2.

Para a caracterização dos conglomerados de obesidade e não obesidade foi realizada a análise descritiva das variáveis ambientais, por meio de medidas de tendência central e dispersão. As variáveis ambientais foram agregadas de acordo com as AAUBS a que pertenciam os indivíduos incluidos no conglomerado.

As variáveis ambientais não apresentaram distribuição simétrica e foram descritas por meio da mediana e intervalo interquartílico (IQ). Para comparar as diferenças entre as mesmas, foi utilizado o teste Mann-Whitney, considerando como nível de significância $=0,05$.

Para verificar possíveis diferenças entre os indivíduos que pertenciam aos conglomerados de obesos e não obesos, realizou-se uma análise comparativa das características dos indivíduos (sexo, cor, escolaridade, estado civil e idade) nos conglomerados de obesos e não obesos, por meio dos testes de diferenças de proporção e mediana, considerando como nível de significância $=0,05$.

Os dados serão apresentados por meio de figuras e tabelas.

Este estudo foi aprovado pelo Comitê de Ética em Pesquisa da Universidade Federal de Minas Gerais, conforme Resolução no 466/2012 do Conselho Nacional de Saúde ${ }^{18}$.

\section{Resultados}

A amostra deste estudo foi constituída por 5.273 indivíduos, com idade média de 45,5 anos (DP $=16,8$ ).

Observou-se que a prevalência de obesidade foi de 12,4\% (IC95\% = 11,5-13,3), sem diferenças entre os sexos $(\mathrm{p}=0,09)$ : no sexo masculino, a prevalência de obesidade foi de 11,5\% (IC95\% $=10,2-12,9)$ e, no feminino, a prevalência foi de $13,0 \%($ IC95\% $=11,9-14,3)$.

Na Figura 2, observa-se a distribuição heterogênea das prevalências de obesidade de acordo com as 148 AAUBS. A prevalência de obesidade variou entre as 148 AAUBS de 0,0 a 38,5\%.

Os resultados da estatística de varredura espacial scan são apresentados na Figura 3. Verificou-se que a área mais central do município (círculo não tracejado) possui uma aglomeração de pessoas obesas. No entanto, a análise espacial não apresentou forte evidência estatística (p-valor = 0,12 ). Adicionalmente, observou-se que a área correspondente a uma região localizada no leste de Belo Horizonte (círculo tracejado) apresentou um conglomerado de pessoas não obesas. Esse conglomerado foi significativo $(\mathrm{p}$-valor $=0,04)$.

A Tabela 1 apresenta uma análise comparativa das características ambientais nos conglomerados de indivíduos obesos e não obesos, sendo as características significativamente diferentes ( $\mathrm{p}$ $<0,001)$, exceto para a densidade populacional.

Observa-se que o conglomerado de indivíduos não obesos apresentou maior diversidade de estabelecimentos de venda de alimentos e locais para prática de atividade física, além de maior renda da vizinhança e menores taxas de homicídios. 
A Tabela 2 apresenta uma análise comparativa das características dos indivíduos nos conglomerados de obesos e não obesos. Não foram verificadas diferenças significativas entre as variáveis analisadas de acordo com os testes de diferenças de proporção e mediana.

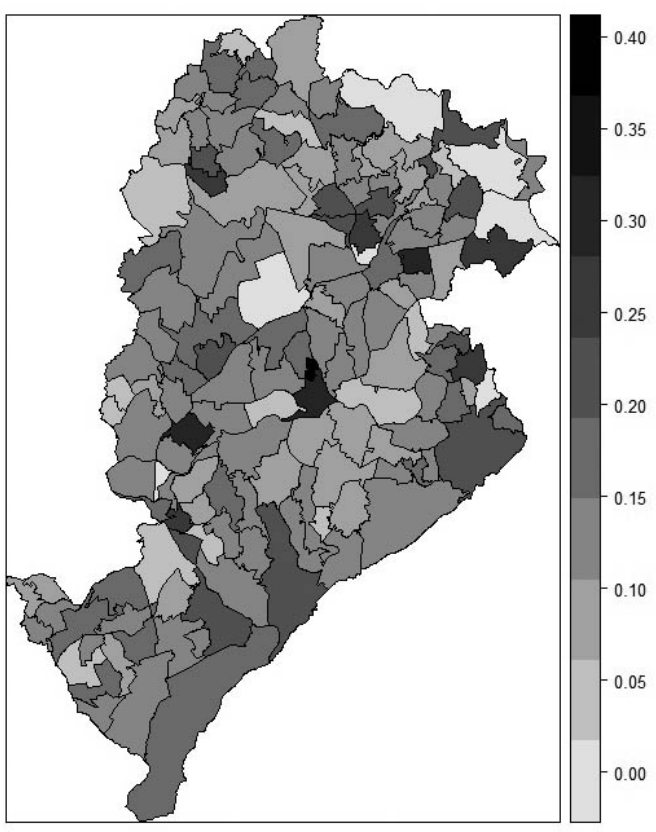

Figura 2. Distribuição espacial das prevalências de obesidade de acordo com as áreas de abrangência das Unidades Básicas de Saúde, Belo Horizonte, 2008-2010.

\section{Discussão}

É crescente o interesse em entender o papel do ambiente urbano sobre o status de saúde e a prevenção de doenças na comunidade, dentre elas a obesidade. Os resultados deste estudo sugerem a presença de um conglomerado significativo de indivíduos não obesos na área urbana. Entretanto, não foi possível mostrar alta evidência significativa de um conglomerado de indivíduos obesos em área central da cidade, o qual apresenta características ambientais significativamente diferentes do conglomerado de não obesos, exceto para a densidade populacional.

Embora a presença de conglomerado de obesidade não tenha apresentado significância estatística, o resultado mostrado pode ser considerado indicador de uma potencial heterogeneidade espacial de obesidade na área de estudo. Neste caso em particular, ausência de significância estatística de aleatoriedade espacial poderia ser explicada pelo número limitado de casos dentro do conglomerado de obesos. Dessa forma, caso novas observações estivessem disponíveis para esse local em particular, seria possível existir maior evidência de ausência aleatoriedade espacial com relação a esse desfecho (rejeição de hipótese de aleatoriedade) e, consequente, delimitação de um conglomerado de obesidade. Na impossibilidade da coleta de novos registros, ao se realizar uma análise comparativa das variáveis no contexto ambiental do conglomerado, verificou-se que a área caracterizada por um conglomerado de obe-

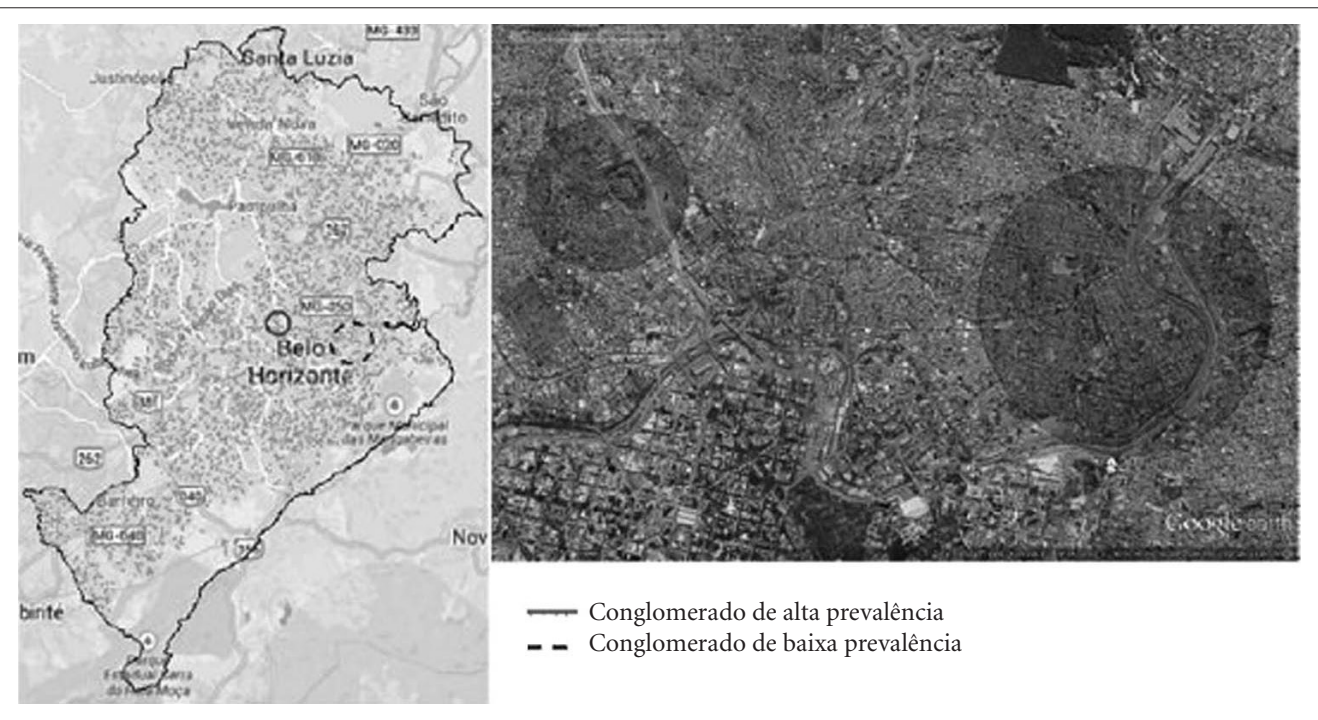

Figura 3. Varredura espacial dos casos obesidade. Belo Horizonte, 2008-2010. 
Tabela 1. Descrição das características ambientais de acordo com os conglomerados de obesos e não obesos. Belo Horizonte, MG. 2008-2010

\begin{tabular}{lccr}
\hline \multicolumn{1}{c}{ Características ambientais } & $\begin{array}{c}\text { Conglomerado de não obesos } \\
\text { Mediana (IQ) }\end{array}$ & $\begin{array}{c}\text { Conglomerado de obesos } \\
\text { Mediana (IQ) }\end{array}$ & $\begin{array}{c}\text { Valor } \\
\text { de p p }\end{array}$ \\
\hline Número de restaurantes & $110(36-110)$ & $28(0-42)$ & $<0,001$ \\
Número de comércios não saudáveis & $195(108-195)$ & $63(2-68)$ & $<0,001$ \\
Número de supermercados e hipermercados & $8(4-8)$ & $0(0-0)$ & $<0,001$ \\
Número de estabelecimentos especializados & $14(9-14)$ & $4,50(1,47)$ & $<0,001$ \\
na venda de frutas e hortaliças & & & $<0,001$ \\
Número de locais para prática de atividade & $30(17-30)$ & $6(0-6)$ & \\
física & & & \\
Densidade populacional (habitantes/km2) & $11.484,73$ & $(6.154,67)$ & 0,784 \\
& $(10.597,09-11.484,73)$ & $18,10(18,10-19,80)$ & $<0,001$ \\
Taxa de homicídio & $6,30(3,23-6,30)$ & 11.600 .000 & $<0,001$ \\
& 75.700 .000 & $(2.685 .892-11.600 .000)$ & $<0,001$ \\
Rendimento total $^{* * *}$ & $(51.400 .000-75.700 .000)$ & $(2.685)$ &
\end{tabular}

Legenda: IQ - Intervalo Interquartílico; ${ }^{*}$ Comércios não saudáveis: bares, lanchonetes e vendedores ambulantes de alimentos; ${ }^{* *}$ Taxa de homicídio por $10000 ;{ }^{* * *}$ Rendimento total nominal mensal de indivíduos com 10 anos ou mais de idade das áreas de abrangência das Unidades Básicas de Saúde.

Tabela 2. Descrição das características individuais de acordo com os conglomerados de obesos e não obesos. Belo Horizonte, MG. 2008-2010.

\begin{tabular}{lcc}
\hline \multicolumn{1}{c}{ Características individuais } & $\begin{array}{c}\text { Conglomerado de não obesos } \\
\mathbf{n}(\%)\end{array}$ & $\begin{array}{c}\text { Conglomerado de obesos } \\
\mathbf{n}(\%)\end{array}$ \\
\hline Sexo & & \\
$\quad$ Masculino & $32(39,51)$ & $10(41,67)$ \\
Feminino & $49(60,49)$ & $14(58,33)$ \\
Cor & & \\
$\quad$ Branca & $44(54,32)$ & $6(25,00)$ \\
Negra & $5(6,17)$ & $3(12,50)$ \\
Parda/Morena & $32(39,51)$ & $15(62,50)$ \\
Escolaridade & & \\
0 a 8 & $16(19,75)$ & $9(37,50)$ \\
9 a 11 & $31(38,27)$ & $11(45,83)$ \\
12 ou mais & $34(41,98)$ & $4(16,67)$ \\
Estado Civil & & \\
Solteiro & $35(44,30)$ & $5(22,73)$ \\
Casado & $29(36,71)$ & $7(31,82)$ \\
Viúvo & $8(10,13)$ & $4(18,18)$ \\
Separado/Divorciado & $7(8,86)$ & $6(27,27)$ \\
Idade & $48(30-63)$ & $51(37-69,75)$
\end{tabular}

* Mediana e Intervalo Interquartílico. Na análise comparativa das características dos indivíduos nos conglomerados de obesos e não obesos não foram verificadas diferenças significativas entre as variáveis analisadas $(\mathrm{p}>0,05)$.

sidade difere estatisticamente da área onde foi encontrada aglomeração de pessoas não obesas. Observou-se menor número de restaurantes, de comércios não saudáveis, de supermercados e hipermercados, de estabelecimentos especializados na venda de frutas e hortaliças e feiras livres e de locais para prática de atividade física nesta área. Verificou-se, ainda, que menor renda total e maior taxa de homicídios foram encontradas na área com maior aglomeração de pessoas obesas. Complementar a esses resultados, foi também mostrado que os resultados de comparações en- 
tre variáveis individuais, como sexo, cor, escolaridade, estado civil e idade não resultaram em diferenças significativas.

Achados de conglomerados espaciais da obesidade foram descritos em alguns estudos de áreas urbanas de populações de Taiwan e norte da América ${ }^{19,20}$. No Canadá, foi observada aglomeração espacial significativa de excesso de peso em todo o país ${ }^{21}$. Entretanto, em área urbana desse mesmo país não foi demonstrado agrupamento com o método de detecção de cluster global $^{22}$. A literatura nessa área mostra que a aplicação de métodos para avaliação de localização espacial de desfechos em saúde e de infraestrutura pode auxiliar na avaliação de intervenções ambientais direcionadas a estímulos à dieta saudável e atividade física ${ }^{23}$. Estudos sugerem que o ambiente alimentar da vizinhança pode influenciar as escolhas alimentares e a ingestão alimentar ${ }^{24,25}$.

Estudo realizado em Detroit mostrou que a presença de grandes lojas de hortifruti na vizinhança associou-se ao consumo de frutas, legumes e verduras, sendo que a presença dos estabelecimentos na vizinhança foi mais importante do que a distância, que não se associou ao consumo ${ }^{26}$. Estudo realizado em Belo Horizonte, demonstrou aumento do escore de consumo de frutas, verduras e legumes à medida que aumentou o número de lojas de hortifruti na vizinhança ${ }^{27}$.

A presença de supermercados, hipermercados e hortifrutis na vizinhança contribui para que as pessoas tenham preços mais acessíveis para a escolha de uma alimentação de melhor qualida$\mathrm{de}^{28}$. Além disso, o maior número de restaurantes pode favorecer melhores escolhas alimentares quando possuem opções alimentares saudáveis ${ }^{29}$. Os fatores socioeconômicos da vizinhança podem influenciar os hábitos de vida, como o comportamento alimentar e a prática de atividade física e, consequentemente, a obesidade ${ }^{30}$.

Estudos mostram que áreas com menor privação socioeconômica apresentam maior densidade de todos os tipos de comércios de alimentos e maior densidade de locais para a prática de esportes $^{31,32}$. Assim, lojas especializadas na venda de alimentos saudáveis, por exemplo, são menos frequentes em vizinhanças mais desfavorecidas e, por isso, alguns alimentos saudáveis estão menos disponíveis ou possuem qualidade inferior em áreas de menor renda ${ }^{28}$.

Em estudos recentes realizados no contexto urbano similar ao deste estudo, foi observado que os estabelecimentos de venda de alimentos estão localizados onde há maior concentração de outros tipos de comércios e serviços e em vi- zinhanças mais favorecidas. Verificou-se, ainda, que o consumo de frutas, verduras e legumes aumenta à medida que a renda total da vizinhança também aumenta ${ }^{27}$.

Com relação ao ambiente social, a insegurança e o aumento da violência nos locais onde as pessoas vivem contribuem para que a prática de atividade física seja dificultada nas grandes cida$\operatorname{des}^{33}$. Ainda que em populações adultas de baixo nível socioeconômico ${ }^{34}$, estudos de ambientes urbanos brasileiros mostram que pessoas com percepção positiva de segurança apresentaram maiores chances de serem ativas no deslocamento. Estudo transversal ${ }^{35}$, realizado com idosos, demonstrou que a boa percepção de segurança durante o dia $(\mathrm{OR}=4,21$; IC95\% = 1,29-13,79), dentre outras variáveis, apresentou associação com a prática de atividade física no lazer nos homens.

Há de se considerar, ainda, algumas limitações no presente estudo. A primeira delas refere-se ao fato de o IMC ser autorrelatado, o que pode se associar com o relato impreciso. Embora o IMC aferido seja ideal, isso não seria econômica e logisticamente viável para um inquérito, como o deste estudo, devido ao caráter prático dos sistemas de vigilância em saúde. Entretanto, esse problema é minimizado por estudos de validação realizados com os participantes da amostra VIGITEL, que demonstraram altos índices de desempenho quando os valores aferidos foram comparados aos autorrelatados e mostraram bons resultados nas análises de sensibilidade e especificidade na caracterização dicotômica do estado nutricional (obeso e não obeso). Limitações adicionais referem-se à amostra, composta de pessoas residentes em domicílios com linha telefônica fixa, e à utilização de dados secundários obtidos de fontes governamentais e comerciais para descrever as características ambientais. Entretanto, o desenho utilizado apresenta aspectos que atestam sua validade em termos de representatividade - desenho aleatório de escolha dos participantes em três anos diferentes do sistema anual de monitoramento de fatores de risco para doenças crônicas. Além disso, foram utilizados métodos espaciais apropriados, que representaram a ausência de componente espacial e a presença de padrão aleatório.

Para o nosso conhecimento, há escassez de trabalhos nacionais que analisem a distribuição espacial de casos de obesidade em adultos em uma cidade de grande porte e que descrevam as características ambientais de acordo com os locais com alta e baixa ocorrência de indivíduos obesos. 
As evidências encontradas neste estudo sugerem que as possíveis razões para a baixa prevalência de obesidade em áreas urbanas brasileiras podem estar relacionadas à melhor organização social e econômica e alta disponibilidade de comércios de alimentos e de locais para a prática de atividade física.

\section{Colaboradores}

FP Matozinhos participou do planejamento da pesquisa, análise estatística, interpretação dos dados, redação do artigo, revisão crítica relevante do conteúdo intelectual e aprovação da versão a ser publicada. CS Gomes e MA Costa participaram da análise estatística, interpretação dos dados, redação do artigo, revisão crítica relevante do conteúdo intelectual e aprovação da versão a ser publicada. LL Mendes e MC Pessoa participaram da análise estatística, redação do artigo, revisão crítica relevante do conteúdo intelectual e aprovação da versão a ser publicada. G Velásquez-Meléndez foi responsável pela concepção e projeto, supervisão, redação do artigo, revisão crítica do conteúdo intelectual e aprovação da versão a ser publicada.

\section{Agradecimentos}

Agradecemos ao Ministério da Saúde pelo apoio financeiro para a realização deste estudo.

\section{Referências}

1. Egger G, Swinburn B. An "ecological” approach to the obesity pandemic. BMJ 1997; 315(7106):477-480.

2. Swinburn B, Egger G, Raza F. Dissecting obesogenic environments: the development and application of a framework for identifying and prioritizing environmental interventions for obesity. Prev Med 1999; 29(6):563-570.

3. Booth KM, Pinkston MM, Poston WS. Obesity and the built environment. J Am Diet Assoc 2005; 105(5):110117.

4. Duncan DT, Castro MC, Gortmaker SL, Aldstadt J, Melly SJ, Bennett GG. Racial differences in the built environment-body mass index relationship? A geospatial analysis of adolescents in urban neighborhoods. Int J Health Geogr 2012; 26(11):11.

5. Ewing R, Brownson RC, Berrigan D. Relationship between urban sprawl and weight of United States youth. Am J Prev Med 2006; 31(6):464-474.

6. Feng J, Glass TA, Curriero FC, Stewart WF, Schwartz BS. The built environment and obesity: a systematic review of the epidemiologic evidence. Health Place 2010; 16(2):175-190.

7. Gilliland JA, Rangel CY, Healy MA, Tucker P, Loebach JE, Hess PM, He M, Irwin JD, Wilk P. Linking childhood obesity to the built environment: a multi-level analysis of home and school neighbourhood factors associated with body mass index. Can J Public Health 2012; 103(9):15-21.

8. Hollands S, Campbell MK, Gilliland J, Sarma S. A spatial analysis of the association between restaurant density and body mass index in Canadian adults. Prev Med 2013; 57(4):258-264.

9. Glaz J, Pozdnyakov V, Wallenstein S. Scan Statistics: Methods and Applications. Boston: Birkhouser Publishers; 2009.

10. Chaix B, Méline J, Duncan S, Jardinier L, Perchoux C, Vallée J, Merrien C, Karusisi N, Lewin A, Brondeel R, Kestens Y. Neighborhood environments, mobility, and health: towards a new generation of studies. Rev Epidemiol Sante Publique 2013; 61(3):139-145.

11. Brasil. Ministério da Saúde (MS). Secretaria de Vigilância em Saúde. Fundação Oswaldo Cruz. Introdução à Estatística Espacial para a Saúde Pública. Brasília: MS; 2007. 
12. Faria RM, Bortolozzi A. Espaço, território e saúde: contribuições de Milton santos para o tema da geografia da saúde no Brasil. Ra’e Ga 2009; 17:31-41.

13. Jacquez GM, Goovaerts P. The emerging role and benefits of boundary analysis in spatiotemporal epidemiology and public health. Spat Spatiotemporal Epidemiol 2010; 1(4):197-200.

14. Reidpath DD, Burns C, Garrard J, Mahoney M, Townsend M. An ecological study of the relationship between social and environmental determinants of obesity. Health Place 2002; 8(2):141-145.

15. Drewnowski A, Rehm CD, Solet D. Disparities in obesity rates: analysis by ZIP code area. Soc Sci Med 2007; 65(12):2458-2463.

16. World Health Organization (WHO). Physical status: the use and interpretation of anthropometry. Geneva: WHO; 1995.

17. Kulldorff M. A spatial scan statistic. Communications in Statistics: Theory and Methods 1997; 26(6):1481-1496.

18. Brasil. Ministério da Saúde (MS). Conselho Nacional de Saúde. Resolução no 466, de 12 de dezembro de 2012. Diário Oficial da União 2013; 13 jun.

19. Chen DR, Wen, TH. Elucidating the changing socio -spatial dynamics of neighborhood effects on adult obesity risk in Taiwan from 2001 to 2005. Health Place 2010; 16(6):1248-1258.

20. Huang L, Tiwari RC, Pickle LW, Zou Z. Covariate adjusted weighted normal spatial scan statistics with applications to study geographic clustering of obesity and lung cancer mortality in the United States. Stat Med 2010; 29(23):2410-2422.

21. Pouliou T, Elliott SJ. An exploratory spatial analysis of overweight and obesity in Canada. Prev Med 2009; 48(4):362-367.

22. Schuurman N, Peters PA, Oliver LN. Are obesity and physical activity clustered? A spatial analysis linked to residential density. Obesity (Silver Spring) 2009; 17(12):2202-2209.

23. Dalton AM, Jones A, Ogilvie D, Petticrew M, White M, Cummins $S$. Using spatial equity analysis in the process evaluation of environmental interventions to tackle obesity: the healthy towns programme in England. Int $J$ Equity Health 2013; 12:43.

24. Bovell-Benjamin AC, Hathorn CS, Ibrahim S, Gichuhi PN, Bromfield EM. Healthy food choices and physical activity opportunities in two contrasting Alabama cities. Health Place 2009; 15(2):429-438.

25. Mason KE, Bentley RJ, Kavanagh AM. Fruit and vegetable purchasing and the relative density of healthy and unhealthy food stores: evidence from an Australian multilevel study. J Epidemiol Community Health 2013; 67(3):231-236.
26. 26. Zenk SN, Lachance LL, Schulz AJ, Mentz G, Kannan S, Ridella W. Neighbourhood retail food environment and fruit and vegetable intake in a multiethnic urban population, subpopulations. Am J Health Promot 2009; 23(4):255-264.

27. Pessoa MC. Ambiente alimentar e consumo de frutas, legumes e verduras em adultos de Belo Horizonte - MG. Belo Horizonte: Universidade Federal de Minas Gerais; 2013.

28. Kamphuis CB, Giskes K, de Bruijn GJ, Wendel-Vos W, Brug J, van Lenthe FJ. Environmental determinants of fruit and vegetable consumption - a systematic review. Br J Nutr 2006; 96(4):620-635.

29. Boone-Heinonen J, Gordon-Larsen P, Kiefe CI, Shikany JM, Lewis CE, Popkin BM. Fast Food Restaurants and Food Stores- Longitudinal associations with diet in young middle-aged adults: The CARDIA Study. Arch Intern Med 2011; 171(13):1162-1170.

30. Thornton LE, Pearce JR, Macdonald L, Lamb KE, Ellaway A. Does the choice of neighbourhood supermarket access measure influence associations with individual-level fruit and vegetable consumption? A case study from Glasgow. Int J Health Geogr 2012; 11:29.

31. Jaime PC, Duran AC, Sarti FM, Lock K. Investigating environmental determinants of diet physical activity, and overweight among adults in Sao Paulo, Brazil. $J$ Urban Health 2011; 88(3):567-581.

32. Pearce J, Hiscock R, Blakely T, Witten K. The contextual effects of neighbourhood access to supermarkets and convenience stores on individual fruit and vegetable consumption. J Epidemiol Community Health 2008; 62(3):198-201.

33. Ewing R, Cervero R. Travel and the built environment: a synthesis. Transportation Research Record 2001; 1780:87-114.

34. Florindo AA, Salvador EP, Reis RS, Guimarães VV. Percepção do ambiente e prática de atividade física em adultos residentes em região de baixo nível socioeconômico. Rev Saude Publica 2011; 45(2):302-310.

35. Salvador EP, Florindo AA, Reis RS, Costa EF. Percepção do ambiente e prática de atividade física no lazer entre idosos. Rev Saude Publica 2009; 43(6):972-980.

Artigo apresentado em 25/09/2014

Aprovado em 29/01/2015

Versão final apresentada em 31/01/2015 
Ciência \& Saúde Coletiva

volume 20 número 9 - 2015

p. 2779

onde se lê:

Fernanda Penido Matozinhos ${ }^{1}$

Gustavo Velásquez Meléndez ${ }^{1}$

Milene Cristine Pessoa ${ }^{2}$

Larissa Mendes ${ }^{3}$

Crizian Saar Gomes ${ }^{1}$

Marcelo Azevedo Costa ${ }^{4}$

leia-se:

Fernanda Penido Matozinhos ${ }^{1}$

Crizian Saar Gomes ${ }^{1}$

Marcelo Azevedo Costa ${ }^{2}$

Larissa Loures Mendes ${ }^{3}$

Milene Cristine Pessoa ${ }^{4}$

Gustavo Velasquez-Melendez ${ }^{1}$

onde se lê:

database for 2008 to 2010 of the Surveillance of Risk and Protective Factors for Chronic Diseases.

leia-se:

database Surveillance of Risk and Protective Factors for Chronic Diseases (Vigitel).

onde se lê:

Proteção para Doenças Crônicas por Inquérito Telefônico de 2008 a 2010.

leia-se:

Proteção para Doenças Crônicas por Inquérito Telefônico (Vigitel)

onde se lê:

$30 \mathrm{~kg} / \mathrm{m} 2$

leia-se:

$\geq 30 \mathrm{~kg} / \mathrm{m}^{2}$ onde se lê:

${ }^{2}$ Universidade Federal de Viçosa.

${ }^{3}$ Universidade Federal de Juiz de Fora.

${ }^{4}$ Departamento de Engenharia de Produção, Universidade Federal de Minas Gerais.

leia-se:

${ }^{2}$ Departamento de Engenharia de Produção, Universidade Federal de Minas Gerais.

${ }^{3}$ Departamento de Nutrição, Universidade Federal de Juiz de Fora.

${ }^{4}$ Departamento de Nutrição e Saúde, Universidade Federal de Viçosa.

p. 2780

onde se lê:

Neste sentido, o objetivo do presente estudo foi analisar a distribuição espacial da obesidade em adultos, de 2008 a 2010, no município de Belo Horizonte, Minas Gerais.

leia-se:

Neste sentido, o objetivo do presente estudo foi analisar a distribuição espacial da obesidade em adultos, no município de Belo Horizonte, Minas Gerais.

p. 2781

onde se lê:

menores taxas de homicídios

leia-se:

menor taxa de homicídios 